\title{
Chest Pain Due to Late Huge Coronary Pseudoaneurysm Following Stent Implantation
}

\author{
Marco Caruso ${ }^{1}$, Salvatore Evola ${ }^{1}$, Khalil Fattouch ${ }^{2}$, Umberto M Bracale ${ }^{2}$, Egle Incalcaterra ${ }^{1}$, \\ Eluisa La Franca ${ }^{1}$, Giuseppina Novo ${ }^{1}$, Giuseppe Andolina ${ }^{1}$ and Salvatore Novo ${ }^{1}$
}

\begin{abstract}
A 50-year-old man was referred to our hospital because of persistent atypical chest pain. His past medical history was remarkable for a non ST elevation myocardial infarction, treated five months previously with PCI on the right coronary artery. Two months later, for chest pain, he underwent coronary angiography that showed a right coronary artery with slight ectasia near the stent. Five months later for the persistence of atypical chest pain he came to our clinic. Chest CT showed a $31.5 \mathrm{~mm}$ hematoma of the right coronary artery. Coronary angiography revealed a giant aneurysm, proximal to the stent. In the light of rapid growth of aneurysm, the risk of rupture and symptoms, we decided to treat the aneurysm with covered stents. The patient underwent successful PCI with regression of symptoms.
\end{abstract}

Key words: coronary pseudoaneurysm, PCI, chest pain

(Intern Med 50: 577-579, 2011)

(DOI: 10.2169/internalmedicine.50.4566)

\section{Introduction}

Coronary aneurysm formation is a rare complication after bare metal stent implantation. Open surgical repair has long been the treatment of choice. More recently, endovascular techniques have offered a less invasive approach. The initial reports have been very encouraging, but the best endovascular modality is not yet clear. We report the clinical manifestation, diagnosis and endovascular treatment of a huge coronary pseudoaneurysm after bare metal stent implantation.

\section{Case Report}

We report the case of a 50-year-old man with risk factors for coronary artery disease of ex-smoking, obesity and hyperlipidemia. His past medical history was remarkable for a non-ST elevation myocardial infarction, treated five months previously with percutaneous coronary intervention (PCI) and bare metal stent (BMS) $(4 \times 19 \mathrm{~mm})$ implantation of the right coronary artery (RCA). Two months later he developed atypical chest pain and underwent coronary angiography that showed a normal left coronary artery (LCA) and a RCA

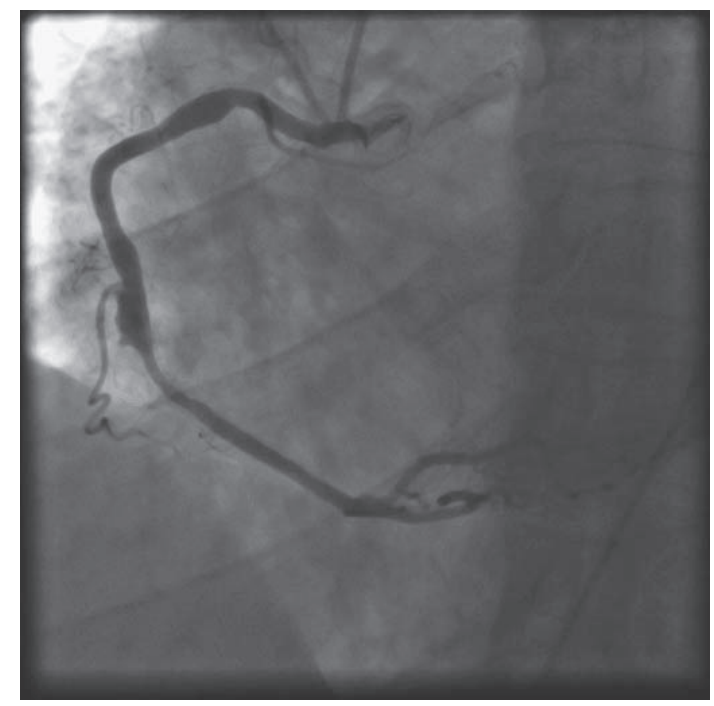

Figure 1. Angiography showing right coronary artery with slight ectasia two months after PCI.

with slight ectasia over the proximal and mid segment and a moderate (50\%) stenosis after the stent (Fig. 1). Five months later with the complaint of persistent atypical chest pain he

${ }^{1}$ Cardiology Unit, University of Palermo, Italy and ${ }^{2}$ Department of Surgical and Oncological Science, University of Palermo, Italy Received for publication September 21, 2010; Accepted for publication November 29, 2010 Correspondence to Dr. Marco Caruso, marcar@unipa.it 


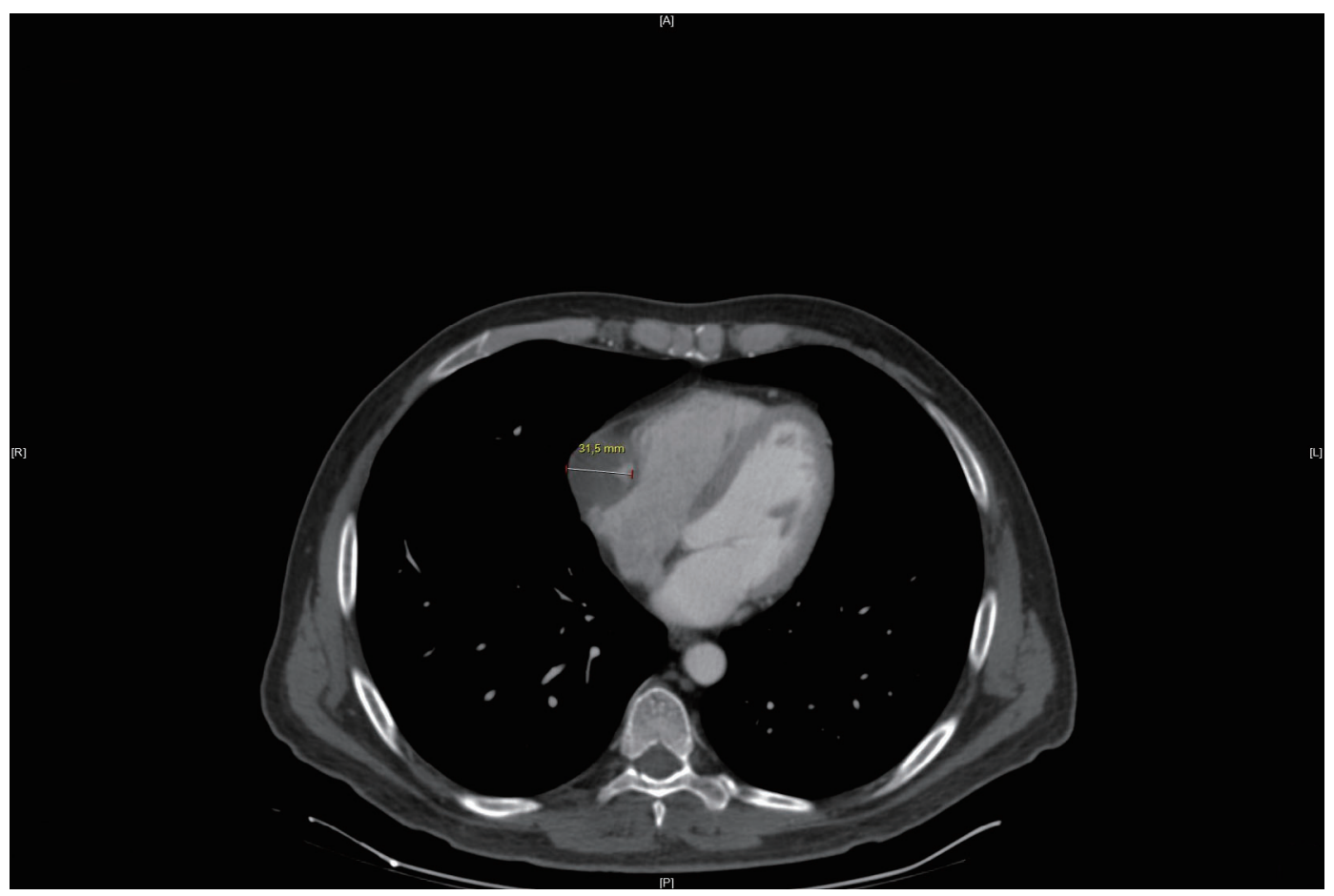

Figure 2. Chest CT showing right coronary hematoma near the stent.

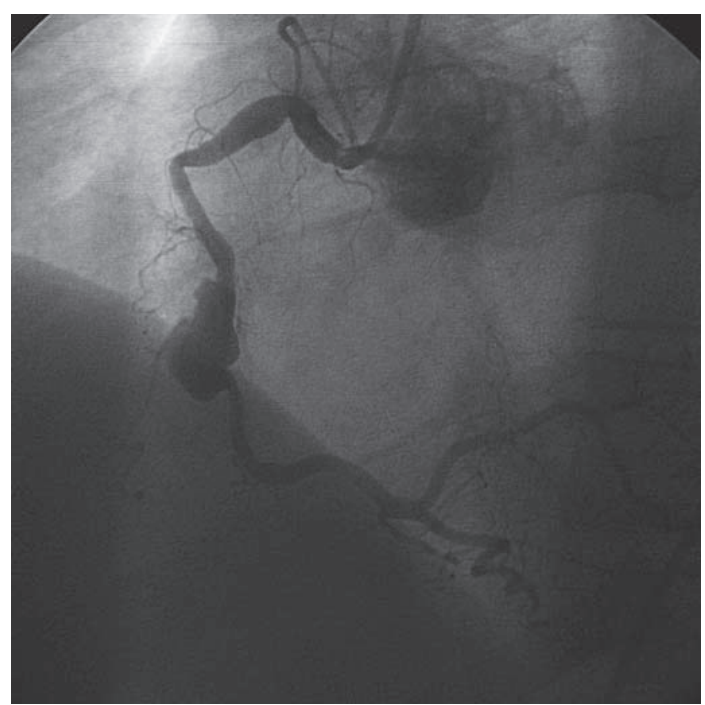

Figure 3. Angiography showing right coronary artery with a huge psedoaneurysm five months after PCI.

came to our clinic. He was taking aspirin (100 mg/day). His troponin was normal, with normal full blood count, electrolytes and renal function. ECG showed no new changes. Chest X-ray was normal. Subsequent chest computed tomography showed a $31.5 \mathrm{~mm}$ hematoma of the right coronary artery, near the stent (Fig. 2). Coronary angiography revealed a giant aneurysm, proximal to the stent (Fig. 3). In the light of rapid growth of aneurysm, of the risk of rupture and symptoms we decided to treat the aneurysm with covered stents.

We performed PCI deploying two polytetrafluroethylene (PTFE) graft covered stents (Jostent Graftmaster, Abbott

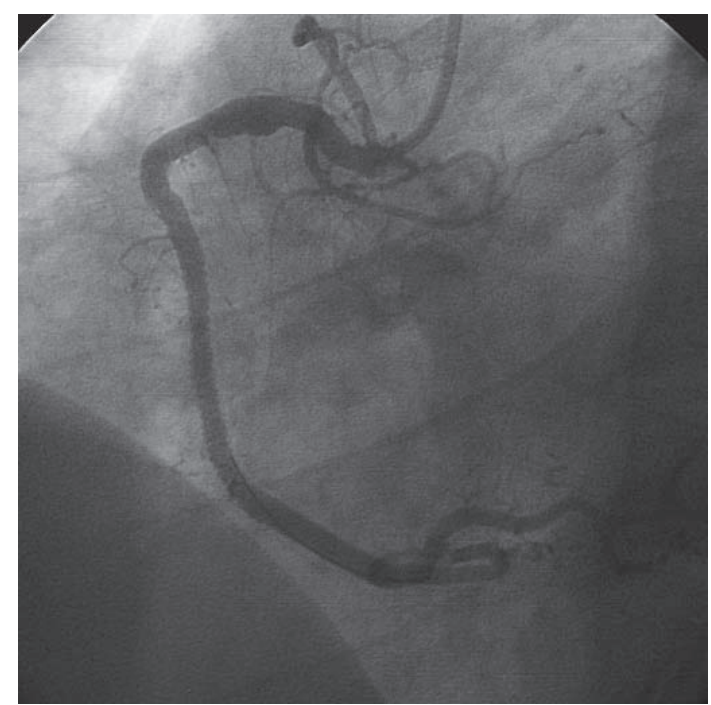

Figure 4. Right coronary artery after PCI with two PTFE covered stents and one DES.

Vascular, Abbott Park, IL, USA; $3 \times 19 \mathrm{~mm}$ and $3 \times 16 \mathrm{~mm}$ ) to tamponade pseudoaneurysm. Because of a leak of contrast media, proximal to the two covered stents, we implanted an everolimus eluting stent (Xience V, Abbott Laboratories, Abbott Park, IL, USA; $3.5 \times 12 \mathrm{~mm}$ ) in overlap with the proximal covered stent. There was an excellent final result without any residue leak of contrast media, with regression of symptoms (Fig. 4). At discharge dual antiplatelet therapy for at least 1 year was recommended (100 mg of aspirin and $75 \mathrm{mg}$ of clopidogrel daily). At the two-week follow-up visit the patient was asymptomatic. A follow-up visit and a noninvasive coronary angiography by computed 
tomography has been recommended at 6 months.

\section{Discussion}

Coronary artery aneurysms are angiographically defined as localized luminal dilatations measuring at least 1.5-2 times the diameter of a normal adjacent reference segment (1). A true aneurysm is defined by IVUS as a segment with a maximal lumen area larger than a proximal reference lumen by at least $50 \%$ with the presence of a wall containing intima, media and adventitia. In contradistinction, a pseudoaneurysm results from the loss of vessel wall integrity and transition from a three-layered wall to an outwardly bulging monolayer. Pseudoaneurysm usually occurs after PCI as a result of traumatic dissection or perforation of a coronary artery resulting in the disruption of the media without blood seepage through the adventitia or by deep resection of the vessel wall with directional atherectomy $(2,3)$. Moreover, there have been reports of DES fracture with pseudoaneurysm formation (4). On the other hand, true coronary aneurysm formation is a rare complication after DES implantation, due to late acquired stent malapposition (LASMA) $(5,6)$.

In the present case we have no IVUS images, but angiographic findings (site of aneurysm proximal to the stent) and CT images led us to suspect a pseudoaneurysm subsequent to previous PCI. The peculiarity of the present case is the presence of a documentation of angiographic progression of the pseudoaneurysm (Fig. 1 and Fig. 3), moreover, to our knowledge there are no cases of such a large pseudonaurysm.

Another point of interest is relative to symptoms: pseudoaneurysm after PCI are generally discovered during subsequent angiography when patients present with recurrent angina. The present patient suffered atypical chest pain without electrocardiographic signs of ischemia or troponin elevation. It is possible that the large pseudoaneurysm caused pleuritic pain. So after PCI each chest pain should be well evaluated, in fact pseudoaneurysm which developed after PCI have been associated with an adverse outcome when left untreated (7) because of catastrophic complications such as fissuration in the pericardium (8) or left ventricular inflow obstruction (9).

There is no standard therapy recommended for pseudoaneurysm occurring after PCI. Some patients underwent CABG with resection of the pseudoaneurysm (3). Other investigators suggested the use of vein graft-coated stent (8) or polytetrafluroethylene (PTFE) graft-covered stents (10). Another case has been successfully treated with IVUSguided primary stenting (2). In the present case we evaluated all options. We excluded medical therapy because of the significant expansion of the pseudoaneurysm over time and because of symptoms. Taking into account that this patient presented a single vessel disease and that data from the literature encourage the use of PTFE-covered stents in these cases, in accordance with surgeons, we decided to treat the pseudoaneurysm using a covered stent. Surgical approach should have been utilized in case of failure of the invasive procedure.

After the deployment of the two covered stents, a leak of contrast media was noted. For this reason we implanted a DES. These stents have more potential to inhibit the healing process of injured vessel wall and to allow aneurysm formation to progress when compared with BMS. We chose to implant a DES to reduce the risk of restenosis, which is higher after PCI with covered stents. In addition, the lesion was a pseudoaneurysm, which has a different pathogenetic mechanism.

To evaluate the long-term clinical outcome, we performed a visit and recommended a noninvasive coronary angiography by computed tomography to assess coronary stent patency and regression of the pseudoaneurysm.

\section{The authors state that they have no Conflict of Interest (COI).}

\section{References}

1. Robinson FC. Aneurysms of the coronary arteries. Am Heart J 109: 129-135, 1985.

2. Kitzis I, Kornowski R, Miller HI. Delayed development of a pseudoaneurysm in the left circumflex artery following angioplasty and stent placement, treated with intravascular ultrasound-guided stenting. Cathet Cardiovasc Diagn 42: 51-53, 1997.

3. Schobel WA, Voelker W, Haase KK, Karsch KR. Occurrence of a saccular pseudoaneurysm formation two weeks after perforation on the left anterior descending coronary artery during balloon angioplasty in acute myocardial infarction. Cathet Cardiovasc Interv 47: 341-346, 1999.

4. Okamura T, Hiro T, Fujii T, et al. Late giant coronary aneurysm associated with a fracture of sirolimus eluting stent: a case report. J Cardiol 51: 74-79, 2008.

5. Ruin-Nodar JM, Valencia J, Pineda J. Coronary aneurysm after drug-eluting stents implantation. Eur H Journal 28: 2826, 2007.

6. Zbinden R, Eshtehardi P, Cook S. Coronary aneurysm formation in a patient early after everolimus-eluting stent implantation. J Invasive Cardiol 20: E174-E175, 2008.

7. Maehara A, Mintz GS, Castagna MT, et al. Intravascular ultrasound assessment of spontaneous coronary artery dissection. Am J Cardiol 89: 466-468, 2002.

8. Di Mario C, Caprari M, Santoli C, et al. Transcatheter repair of a large coronary pseudoaneurysm using ultrasound guidance and vein-covered stents. G Ital Cardiol 27: 701-705, 1997.

9. Shao JH, Aronow WS, Kupersmith A, et al. Pseudoaneurysm with thrombus and left ventricular inflow obstruction after left circumflex stenting. Am J Ther 15: 180-183, 2008.

10. Nameki M, Ishiwata S, Momomura S. Large pseudoaneurysm after left main trunk stenting sealed by polytetrafluorethylenecovered stent. Catheter Cardiovasc Interv 60: 233-235, 2003.

(C) 2011 The Japanese Society of Internal Medicine http://www.naika.or.jp/imindex.html 